\title{
RATIONAL HOMOTOPY TYPE OF THE CLASSIFYING SPACE FOR FIBREWISE SELF-EQUIVALENCES
}

\author{
URTZI BUIJS AND SAMUEL B. SMITH
}

(Communicated by Brooke Shipley)

\begin{abstract}
Let $p: E \rightarrow B$ be a fibration of simply connected CW complexes with finite base $B$ and fibre $F$. Let aut $_{1}(p)$ denote the identity component of the space of all fibre-homotopy self-equivalences of $p$. Let $\operatorname{Baut}_{1}(p)$ denote the classifying space for this topological monoid. We give a differential graded Lie algebra model for $\operatorname{Baut}_{1}(p)$, connecting the results of recent work by the authors and others. We use this model to give classification results for the rational homotopy types represented by $\operatorname{Baut}_{1}(p)$ and also to obtain conditions under which the monoid $\operatorname{aut}_{1}(p)$ is a double loop-space after rationalization.
\end{abstract}

\section{INTRODUCTION}

We consider a variation on the following classical problem for principal bundles. Fix a space $B$ and group $G$. Given a principal $G$-bundle $p: E \rightarrow B$ let $\mathcal{G}(p)$ denote the gauge group consisting of $G$-equivariant automorphisms of $p$ as studied in [1. The gauge group classification problem is that of determining the distinct $H$ homotopy types represented by the groups $\mathcal{G}(p)$ for fixed $B$ and $G$ (see, for example, [16, 5, 17). Refining this problem is the problem of determining the homotopy types represented by the classifying spaces $\mathrm{B} \mathcal{G}(p)$ (see, for example, 5, 17, 24]). In this connection, we have the identity $\mathrm{BG}(p) \simeq \operatorname{map}(B, B G ; h)$, where $h: B \rightarrow B G$ is the classifying map [12, 1].

The complexity of the gauge group classification problem is strictly a torsion phenomenon. After rationalization, the gauge groups are all $H$-homotopy commutative and pairwise $H$-homotopy equivalent when $B$ is a compact metric space and $G$ is a connected Lie group [15, Th. D]. Further, the classifying spaces $B \mathcal{G}(p)$ are pairwise homotopy equivalent for $B$, a finite CW complex (see Remark 3.4.3, below).

A related classification problem with rich rational homotopy theory is obtained by moving to the setting of fibrations $p: E \rightarrow B$ with fibre $F$. Here the analogue of the gauge group is the monoid $\operatorname{aut}_{1}(p)$ of fibrewise self-equivalences homotopic to the identity. Passing to the Dold-Lashof classifying space [7, for $B$ simply connected, we have the identity

$$
\operatorname{Baut}_{1}(p) \simeq \widetilde{\operatorname{map}}\left(B, \operatorname{Baut}_{1}(F) ; h\right) .
$$

Received by the editors July 26, 2011 and, in revised form, September 18, 2011.

2010 Mathematics Subject Classification. Primary 55P62, 55Q15.

Key words and phrases. Fibre-homotopy equivalences, classifying space, Quillen model, function space, Lie derivation.

The first author was partially supported by the Ministerio de Ciencia e Innovación grant MTM2010-15831 and by the Junta de Andalucía grant FQM-213. 
Here $h: B \rightarrow \operatorname{Baut}_{1}(F)$ is the classifying map of the fibration $p$ and $\widetilde{\text { map }}$ denotes the universal cover of the function space by [4] (see Proposition 2.1, below). Unlike the gauge groups, the monoids aut ${ }_{1}(p)$ and their classifying $\operatorname{spaces} \operatorname{Baut}_{1}(p)$ display nonequivalent rational homotopy types for fixed $F$ and $B$. This is true even in the case of principal bundles. For instance, we have:

Example 1.1. The classifying spaces $\operatorname{Baut}_{1}(p)$ for principal $S U(n)$-bundles $p$ over $\mathbb{C} P^{m}$ represent $\max \{1, n-1, m\}$ distinct rational homotopy types.

The monoid $\operatorname{aut}_{1}(p)$ is not often $H$-homotopy commutative after rationalization. For example, when $p$ is a principal $S U(n)$-bundle over $\mathbb{C} P^{m}$, as above, $\operatorname{aut}_{1}(p)$ is never $H$-homotopy commutative after rationalization for $n \geq 4$. On the other hand, there are a number of interesting cases in which $\operatorname{aut}_{1}(p)$ is, in fact, $H$-equivalent to a double loop-space after rationalization, i.e., $\operatorname{Baut}_{1}(p)$ is a rational $H$-space. We mention one here:

Example 1.2. Let $p: E \rightarrow B$ be a principal $G$-bundle with decomposable rational characteristic classes where $G$ is a simply connected Lie group and $B$ is a finite, simply connected CW complex. Let $J=\operatorname{Hur}_{E} \circ j_{\sharp}: \pi_{*}(G) \rightarrow H_{*}(E ; \mathbb{Q})$, where $j: G \rightarrow E$ is the fibre inclusion, $j_{\sharp}: \pi_{*}(G) \rightarrow \pi_{*}(E)$ is the induced map and $\operatorname{Hur}_{E}: \pi_{*}(E) \rightarrow H_{*}(E ; \mathbb{Q})$ is the rational Hurewicz map. If $E$ is formal and $J=0$, then $\operatorname{Baut}_{1}(p)$ is a rational $H$-space.

We deduce Examples 1.1 and 1.2 and related results as a consequence of a differential graded (DG) Lie algebra model or Quillen model for the space $\operatorname{Baut}_{1}(p)$. Our starting point is the description of the rational Samelson Lie algebra of $\operatorname{aut}_{1}(p)$ given in [9] which, in turn, extends the famous model for $\operatorname{Baut}_{1}(F)$ described by Sullivan [22]. Let $p: E \rightarrow B$ be a fibration of simply connected CW complexes. Then $p$ admits a relative minimal model which is an injection of DG algebras $I: \mathcal{B}, d_{B} \rightarrow \mathcal{B} \otimes \Lambda W, D$ (see [8, Prop. 15.6]). Let $\operatorname{Der}_{\mathcal{B}}^{n}(\mathcal{B} \otimes \Lambda W)$ denote the vector space of all linear self-maps $\theta$ of $\mathcal{B} \otimes \Lambda W$ reducing degrees by $n$ and satisfying $\theta(a b)=\theta(a) b-(-1)^{n|a|} a \theta(b)$ and $\theta(\mathcal{B})=0$. The graded space $\operatorname{Der}_{\mathcal{B}}(\mathcal{B} \otimes \Lambda W)$ then has the structure of a DG Lie algebra with the commutator bracket $\left[\theta_{1}, \theta_{2}\right]=\theta_{1} \circ \theta_{2}-(-1)^{\left|\theta_{1}\right|\left|\theta_{2}\right|} \theta_{2} \circ \theta_{1}$ and differential $\mathcal{D}(\theta)=[D, \theta]$. When $E$ and $B$ are finite, [9, Th. 1] gives an isomorphism of graded Lie algebras

$$
\pi_{*}\left(\operatorname{aut}_{1}(p)\right) \otimes \mathbb{Q},[,] \cong H_{*}\left(\operatorname{Der}_{\mathcal{B}}(\mathcal{B} \otimes \Lambda W)\right),[,],
$$

where the former space has the Samelson bracket. We refine the above isomorphism with our main result.

Define the connected DG Lie algebra $\operatorname{Der}_{\mathcal{B}}(\mathcal{B} \otimes \Lambda W), \mathcal{D}$ by:

$$
\mathcal{D e r}_{\mathcal{B}}^{n}(\mathcal{B} \otimes \Lambda W)=\left\{\begin{array}{cc}
\operatorname{Der}_{\mathcal{B}}^{n}(\mathcal{B} \otimes \Lambda W) & \text { for } n \geq 2 \\
Z \operatorname{Der}_{\mathcal{B}}^{1}(\mathcal{B} \otimes \Lambda W) & \text { for } n=1
\end{array}\right.
$$

Here $Z$ means the space of cycles. The bracket and differential $\mathcal{D}$ are those described above.

Theorem 1. Let $p: E \rightarrow B$ be a fibration of simply connected finite type $C W$ complexes with fibre $F$. Suppose $B$ and $F$ are finite. Then $\mathcal{D e r}_{\mathcal{B}}(\mathcal{B} \otimes \Lambda W), \mathcal{D}$ is a Quillen model for $\operatorname{Baut}_{1}(p)$.

The paper is organized as follows. In Section 2, we prove Theorem 1 Our proof is based on the main result of [2] which gives a Quillen model for function space 
components. We construct an explicit isomorphism between the DG Lie algebra $\mathcal{D e r}_{\mathcal{B}}(\mathcal{B} \otimes \Lambda W), \mathcal{D}$ and this model for the component map $\left(B, \operatorname{Baut}_{1}(F) ; h\right)$. We then invoke the identity (11). In Section 3, we apply Theorem 1 to deduce a number of applications along the lines of Examples 1.1 and 1.2. We assume familiarity with rational homotopy theory as in the text 8$]$.

\section{A Quillen model For $\operatorname{Baut}_{1}(p)$}

Fix a fibration $p: E \rightarrow B$ with fibre $F$ as in Theorem 1. That is, all spaces are simply connected, finite type CW complexes with $B$ and $F$ finite. Define $\operatorname{aut}_{1}(p)$ to be the path-component of the identity map in the fibrewise function space $\operatorname{map}_{B}(E, E)$ consisting of maps $f: E \rightarrow E$ satisfying $p \circ f=p$. Here $\operatorname{map}_{B}(E, E)$ is topologized as a subspace of $\operatorname{map}(E, E)$. Then, with our hypotheses, $\operatorname{aut}_{1}(p)$ is a strictly associative, homotopy inversive $H$-space of CW type (see [9, Prop. 2.3]) and

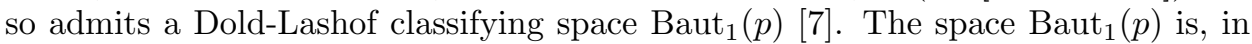
turn, a simply connected CW complex and so admits a rationalization $\operatorname{Baut}_{1}(p)_{\mathbb{Q}}$. Our main result, Theorem 1, determines the homotopy type of $\operatorname{Baut}_{1}(p)_{\mathbb{Q}}$.

Our proof of Theorem 1 depends on three results, which we discuss now. First off, we deduce the identity (1) as a consequence of the main result of [4]. The fibration $p$ above is induced from the universal $F$-fibration by a map $f: B \rightarrow \operatorname{Baut}(F)$ (cf. [18]). Since $B$ is simply connected, $f$ lifts to a map $h: B \rightarrow \operatorname{Baut}_{1}(F)$. We refer to $h$ as the classifying map for $p$.

Proposition 2.1 (Booth-Heath-Morgan-Piccinni). Let $p: E \rightarrow B$ be a fibration with fibre $F$ as hypothesized in Theorem 1. Then there is a homotopy equivalence:

$$
\operatorname{Baut}_{1}(p) \simeq \widetilde{\operatorname{map}}\left(B, \operatorname{Baut}_{1}(F) ; h\right) .
$$

Proof. By [4, Th. 3.3], there is a homotopy equivalence:

$$
\operatorname{Baut}(p) \simeq \operatorname{map}(B, \operatorname{Baut}(F) ; f) .
$$

Since $B$ is simply connected, the inclusion $\operatorname{aut}_{1}(F) \hookrightarrow \operatorname{aut}(F)$ induces a homotopy equivalence $\operatorname{map}\left(B, \operatorname{Baut}_{1}(F) ; h\right) \simeq \operatorname{map}(B, \operatorname{Baut}(F) ; f)$. Thus

$$
\operatorname{Baut}(p) \simeq \operatorname{map}\left(B, \operatorname{Baut}_{1}(F) ; h\right) .
$$

Taking universal covers gives the desired result.

The second two results we need are in rational homotopy theory. We say that a DG Lie algebra $L, \delta$ is a Quillen model for a simply connected CW complex $X$ if there is a quasi-isomorphism $\psi: \mathcal{C}^{*}(L, \delta) \rightarrow A_{\mathrm{PL}}(X ; \mathbb{Q})$. Here $A_{\mathrm{PL}}(X ; \mathbb{Q})$ is the DG algebra of rational polynomial forms on $X$ [8. Sec. 10]. The DG algebra $\mathcal{C}^{*}(L, \delta)$ is the commutative cochain algebra for $L, \delta$ (see [8, Sec. 23]). We recall, as graded algebra $\mathcal{C}^{*}(L, \delta)=\Lambda\left(s^{-1} L^{\sharp}\right)$, the free graded algebra generated by the desuspension of the dual $L^{\sharp}=\operatorname{Hom}(L, \mathbb{Q})$ of $L$. The differential $d$ is given by $d=d_{1}+d_{2}$, where

$$
\left\langle d_{1} s^{-1} z ; s x\right\rangle=-\langle z, \delta x\rangle \quad \text { and } \quad\left\langle d_{2} s^{-1} z ; s x_{1}, s x_{2}\right\rangle=(-1)^{\left|x_{1}\right|}\left\langle z,\left[x_{1}, x_{2}\right]\right\rangle .
$$

Here $z \in L^{\sharp}$ and $x, x_{1}, x_{2} \in L$.

Proposition 2.2 (Sullivan). Let $F$ be a finite, simply connected $C W$ complex. Let $\Lambda W, d$ be the Sullivan minimal model for $F$. The $D G$ Lie algebra $\operatorname{Der}(\Lambda W), \mathcal{D}$ is then a Quillen model for $\operatorname{Baut}_{1}(F)$. 
Proof. Corollary 7.4.4 of [23] gives a Quillen model, for $\operatorname{Baut}_{1}(F)$. By [11, Th. 2], this model is quasi-isomorphic to $\operatorname{Der}(\Lambda W), \mathcal{D}$.

The third result we need is a main result of 2 . Let $h: B \rightarrow Y$ be a map of simply connected $\mathrm{CW}$ complexes with $B$ finite. We say that $\mathcal{B}, d_{B}$ is a $D G$ algebra model for $B$ if there is a quasi-isomorphism from the Sullivan minimal model of $B$ to $\mathcal{B}, d_{B}$. Since $B$ is finite, $B$ admits a finite-dimensional DG algebra model $\mathcal{B}, d_{B}$. Let $L, \delta$ be a Quillen model for $Y$. Then we can choose a DG algebra morphism $\phi: \mathcal{C}^{*}(L, \delta) \rightarrow \mathcal{B}, d_{B}$ modeling $h$. Let $\mathcal{C}_{*}(L, \delta)$ and $\mathcal{B}^{\sharp}, d_{B}^{\sharp}$ denote the DG coalgebras dual to $\mathcal{C}^{*}(L, \delta)$ and $\mathcal{B}, d_{B}$, respectively. Let $\tilde{\phi}$ denote the degree -1 linear map given by the composition:

$$
\tilde{\phi}: \mathcal{B}^{\sharp} \stackrel{\phi^{\sharp}}{\longrightarrow} \mathcal{C}_{*}(L, \delta)=\Lambda s L \stackrel{\xi}{\longrightarrow} s L \stackrel{s}{\longrightarrow} L,
$$

where $\xi$ is the projection onto the indecomposables and $s$ is the suspension isomorphism.

Next, let $\operatorname{Hom}^{n}\left(\mathcal{B}^{\sharp}, L\right)$ denote the space of linear maps lowering degrees by $n$ and $\operatorname{Hom}\left(\mathcal{B}^{\sharp}, L\right)$ the corresponding graded vector space. We equip this graded space with a bracket

$$
\left\{\theta_{1}, \theta_{2}\right\}=[,] \circ\left(\theta_{1} \otimes \theta_{2}\right) \circ \Delta,
$$

where $\Delta$ is the diagonal map and [, ] is the bracket in $L$. We also have the perturbed differential:

$$
D_{\phi} \theta=\delta \theta-(-1)^{|\theta|} \theta d_{B}^{\sharp}+\{\tilde{\phi}, \theta\} .
$$

Truncating as above gives a connected DG Lie algebra $\mathcal{H} o m\left(\mathcal{B}^{\sharp}, L\right), D_{\phi}$ defined by

$$
\mathcal{H o m}{ }^{n}\left(\mathcal{B}^{\sharp}, L\right)=\left\{\begin{array}{cl}
\operatorname{Hom}^{n}\left(\mathcal{B}^{\sharp}, L\right) & \text { for } n>1, \\
Z \operatorname{Hom}^{1}\left(\mathcal{B}^{\sharp}, L\right), & \text { for } n=1 .
\end{array}\right.
$$

We have:

Proposition 2.3 ([2, Th. 10] and [3, Th. 7]). Let $B$ and $Y$ be simply connected $C W$ complexes with $B$ finite. Let $\mathcal{B}, d_{B}$ and $L, \delta$ be a finite-dimensional DG algebra model for $B$ and a Quillen model for $Y$, respectively. Then the DG Lie algebra $\mathcal{H}$ om $\left(\mathcal{B}^{\sharp}, L\right), D_{\phi}$ is a Quillen model for $\widetilde{\operatorname{map}}(B, Y ; h)$.

Returning to the fibration $p: E \rightarrow B$ with hypotheses as in Theorem 1] let $I: \mathcal{B}, d_{B} \rightarrow \mathcal{B} \otimes \Lambda W, D$ be a relative minimal model. Our main result in this section is an isomorphism of DG Lie algebras:

$$
\Psi: \mathcal{D e r}_{\mathcal{B}}(\mathcal{B} \otimes \Lambda W), \mathcal{D} \rightarrow \mathcal{H o m}\left(\mathcal{B}^{\sharp}, \mathcal{H o m}(W, \Lambda W)\right), D_{\varphi} .
$$

Here

$$
\varphi: \mathcal{C}^{*}(\mathcal{D} \operatorname{er}(\Lambda W), \mathcal{D}) \rightarrow \mathcal{B}, d_{B}
$$

is a model for the classifying map $h: B \rightarrow \operatorname{Baut}_{1}(F)$.

We define $\Psi$ to be the restriction to "universal covers" of an isomorphism of graded spaces

$$
\Phi: \operatorname{Hom}(W, \mathcal{B} \otimes \Lambda W) \stackrel{\cong}{\longrightarrow} \operatorname{Hom}\left(\mathcal{B}^{\sharp}, \operatorname{Hom}(W, \Lambda W)\right)
$$

given by

$$
\Phi(\theta)(\beta)(w)=(-1)^{|\theta||\beta|}\left(\beta \otimes \operatorname{id}_{\Lambda W}\right) \theta(w)
$$


Notice that, as graded spaces,

$$
\operatorname{Der}_{\mathcal{B}}(\mathcal{B} \otimes \Lambda W)=\operatorname{Hom}(W, \mathcal{B} \otimes \Lambda W)
$$

and

$$
\operatorname{Hom}\left(\mathcal{B}^{\sharp}, \operatorname{Der}(\Lambda W)\right)=\operatorname{Hom}\left(\mathcal{B}^{\sharp}, \operatorname{Hom}(W, \Lambda W)\right)
$$

with the same identities holding for universal covers.

An alternative description of $\Phi$ is the following: Let $\theta \in \operatorname{Hom}(W, B \otimes \Lambda W)$. Then $\Phi(\theta)$ is the unique map in $\operatorname{Hom}\left(\mathcal{B}^{\sharp}, \operatorname{Hom}(W, \Lambda W)\right)$ making the following diagram commutative:

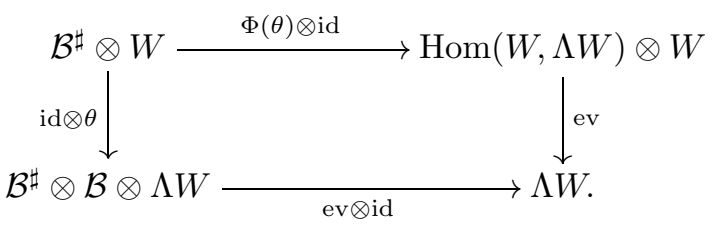

We obtain a related commutative diagram involving the map

$$
\tilde{\varphi}:: \mathcal{B}^{\sharp} \rightarrow \mathcal{D} \operatorname{er}(\Lambda W)
$$

induced by the classifying map $h$. To do so, we observe that $\tilde{\varphi}$ gives rise to a particular choice of a relative minimal model $I: \mathcal{B}, d_{B} \rightarrow \mathcal{B} \otimes \Lambda W, D$ for $p: E \rightarrow B$.

Note that $\tilde{\varphi}$ restricts to give a degree -1 element in $\operatorname{Hom}\left(\mathcal{B}_{+}^{\sharp}, \operatorname{Hom}(W, \Lambda W)\right)$, where $\mathcal{B}_{+}$means elements of positive degree. The differential $D$ in a relative minimal model for $p$ decomposes as

$$
D=d_{W}+d_{T},
$$

where $d_{W}(W) \subseteq \Lambda^{\geq 2} W$ is the differential from the minimal model of the fibre, and $d_{T}(W) \subseteq \mathcal{B}_{+} \otimes \Lambda W$ is the "twisted" part of the differential. Then $d_{T}$ represents a degree -1 element in $\operatorname{Hom}\left(W, \mathcal{B}_{+} \otimes \Lambda W\right)$.

Lemma 2.4. The restriction

$$
\Phi: \operatorname{Hom}\left(W, \mathcal{B}_{+} \otimes \Lambda W\right) \longrightarrow \operatorname{Hom}\left(\mathcal{B}_{+}^{\sharp}, \operatorname{Hom}(W, \Lambda W)\right)
$$

is an isomorphism. Given a classifying map $h: B \rightarrow \operatorname{Baut}_{1}(F)$, we may choose the twisted part of the differential $d_{T}$ in a relative minimal model $I: \mathcal{B}, d_{B} \rightarrow \mathcal{B} \otimes \Lambda W, D$ for $p: E \rightarrow B$ satisfying $\Phi\left(d_{T}\right)=\tilde{\varphi}$. Conversely, given a relative model for $p$ we may choose a model $\varphi$ for $h$ with $\Phi\left(d_{T}\right)=\tilde{\varphi}$.

Proof. That $\Phi$ restricts to an isomorphism is direct. Given a simply connected DG coalgebra $A, \delta$ let $\mathcal{L}^{*}(A, \delta)$ denote the "Quillen construction", a connected DG Lie algebra (see [8, Sec. 22(e)] for details). A DG Lie model for $h$ is then a map $\mathcal{L}(h): \mathcal{L}^{*}\left(\mathcal{B}^{\sharp}, d_{B}^{\sharp}\right) \rightarrow \mathcal{D} \operatorname{er}(\Lambda W), \mathcal{D}$. The map $\tilde{\varphi}$ corresponds here to $\mathcal{L}(h)$ restricted to the basis $\mathcal{B}^{\sharp}$. Next we have a quasi-isomorphism $\psi: \mathcal{D} \operatorname{er}(\Lambda W) \rightarrow \operatorname{cl}\left(\mathcal{L}^{*}\left((\Lambda W)^{\sharp}\right)\right)$, where $\operatorname{cl}\left(\mathcal{L}^{*}\left((\Lambda W)^{\sharp}\right)\right.$ is the DG Lie model for $\operatorname{Baut}_{1}(F)$ given in [23, Ch. 7] and $\psi$ is the DG Lie algebra map constructed in [11, Th. 1]. The composition $\psi \circ$ $\mathcal{L}(h): \mathcal{L}^{*}\left(\mathcal{B}^{\sharp}, d_{B}^{\sharp}\right) \rightarrow \operatorname{cl}\left(\mathcal{L}^{*}\left((\Lambda W)^{\sharp}\right), d_{W}^{\sharp}\right)$ gives rise, via the classification theorem [23. Prop. 7.2(11)], to a split exact sequence of DG Lie algebras of the form

$$
\left.0 \rightarrow \mathcal{L}^{*}\left((\Lambda W)^{\sharp}\right), d_{W}^{\sharp}\right) \rightarrow L, \delta \rightarrow \mathcal{L}^{*}\left(\mathcal{B}^{\sharp}, d_{B}^{\sharp}\right) \rightarrow 0 .
$$

Applying $\mathcal{C}^{*}$ we see that $\mathcal{C}^{*}(L, \delta) \cong \mathcal{B} \otimes \Lambda W, D$ for some differential $D$. We take this as the relative model $I: \mathcal{B}, d_{B} \rightarrow \mathcal{B} \otimes \Lambda W, D$ for $p$. Tracing through the construction, we see that $\Phi\left(d_{T}\right)=\tilde{\varphi}$. The converse result is proved similarly. 
Lemma 2.4 gives the commutativity of the following diagram:

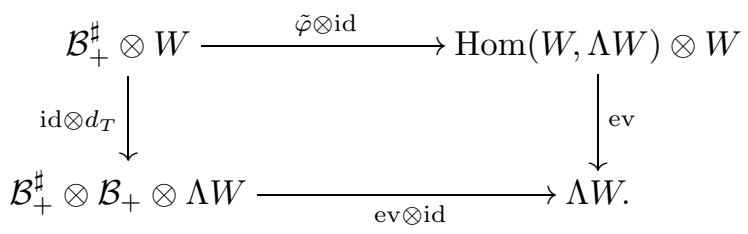

Our main technical result in this section is the following.

Theorem 2.5. Let $\mathcal{B}, d_{B}$ be a finite-dimensional $D G$ algebra and $W$ a graded vector space of finite type. Let $I: \mathcal{B}, d_{B} \rightarrow \mathcal{B} \otimes \Lambda W, D$ be a relative minimal model with twisted differential $d_{T}$. Suppose $\tilde{\varphi}: \mathcal{B}^{\sharp} \rightarrow \operatorname{Hom}(W, \Lambda W)$ gives the commutativity of diagram (6). The restriction

$$
\Psi: \operatorname{Der}_{\mathcal{B}}(\mathcal{B} \otimes \Lambda W), \mathcal{D} \rightarrow \mathcal{H o m}\left(\mathcal{B}^{\sharp}, \mathcal{D} \operatorname{er}(\Lambda W)\right), D_{\varphi}
$$

of the map $\Phi$ defined by (4) is an isomorphism of graded DG Lie algebras.

Proof. Since $\Psi$ is evidently an isomorphism of graded spaces, it suffices to show that $\Psi$ commutes with differentials and brackets.

We first show that

$$
D_{\varphi} \circ \Psi=\Psi \circ \mathcal{D} .
$$

Let $\theta \in \operatorname{Der}_{\mathcal{B}}(\mathcal{B} \otimes \Lambda W), \beta \in \mathcal{B}^{\sharp}, w \in W$. Then we have

$$
\begin{aligned}
\Psi(\mathcal{D} \theta)(\beta)(w)= & (-1)^{(|\theta|+1)|\beta|}\left(\beta \otimes \operatorname{id}_{\Lambda W}\right)\left(D \circ \theta-(-1)^{|\theta|} \theta \circ D\right)(w) \\
= & (-1)^{(|\theta|+1)|\beta|}\left(\beta \otimes i d_{\Lambda W}\right)\left[\left(d_{B} \otimes i d_{\Lambda W}\right) \theta(w)+\left(i d_{\mathcal{B}} \otimes d_{T}\right) \theta(w)\right. \\
& \left.+\left(i d_{\mathcal{B}} \otimes d_{W}\right) \theta(w)+(-1)^{|\theta|+1} \theta d_{T} w+(-1)^{|\theta|+1} \theta d_{W} w\right] \\
= & (a)+\left(b^{\prime}\right)+\left(c^{\prime}\right)+\left(b^{\prime \prime}\right)+\left(c^{\prime \prime}\right) .
\end{aligned}
$$

We see that $(b)=\left(b^{\prime}\right)+\left(b^{\prime \prime}\right)$ and $(c)=\left(c^{\prime}\right)+\left(c^{\prime \prime}\right)$.

On the other hand,

$$
\begin{aligned}
D_{\varphi} \Psi(\theta)(\beta)(w) & =\left(\mathcal{D} \circ \Psi(\theta)+(-1)^{|\theta|+1} \Psi(\theta) \circ d_{B}^{\sharp}+\operatorname{ad}_{\varphi} \Psi(\theta)\right)(\beta)(w) \\
& =(C)+(A)+(B) .
\end{aligned}
$$

A straightforward computation using the commutativity of the following diagrams:
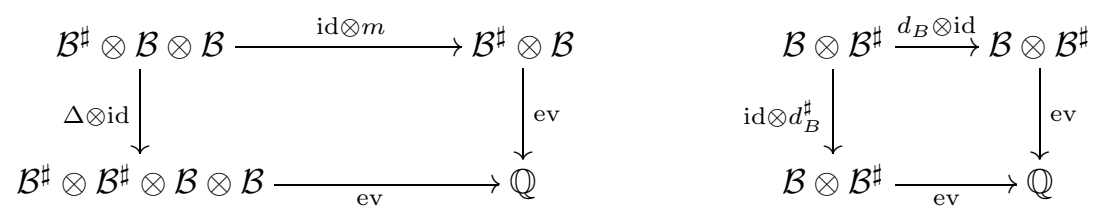

and the diagrams (5), (6) gives $(a)=(A),(b)=(B),(c)=(C)$.

For instance, to see explicitly the equality $(b)=(B)$, it is enough to show that the following compositions agree:

$(\mathrm{ev} \otimes \mathrm{id}) \circ\left(\mathrm{id}_{\mathcal{B}^{\sharp}} \otimes\left(m \otimes \mathrm{id}_{\Lambda W}\right) \circ\left(\left(\mathrm{id}_{\mathcal{B}^{\sharp}} \otimes d_{T}\right) \circ \theta+(-1)^{|\theta|} \theta \circ d_{T}\right)\right): \mathcal{B}^{\sharp} \otimes W \rightarrow \Lambda W$,

$$
\mathrm{ev} \circ\left(\operatorname{ad}_{\varphi} \Psi(\theta) \otimes \operatorname{id}_{\Lambda W}\right): \mathcal{B}^{\sharp} \otimes W \rightarrow \Lambda W .
$$

Note that the first composition splits into two summands. Recall that $\operatorname{ad}_{\varphi}(f)=$ $[,] \circ(\varphi \otimes f) \circ \Delta$, where the bracket [, ] in $\operatorname{Der}(\Lambda W)$ is the commutator. Hence the second composition also splits into two summands. Then, the first summand of both compositions appears as the two possible exterior compositions from the 
top left corner to the bottom right corner of the following commutative diagram, so they agree:

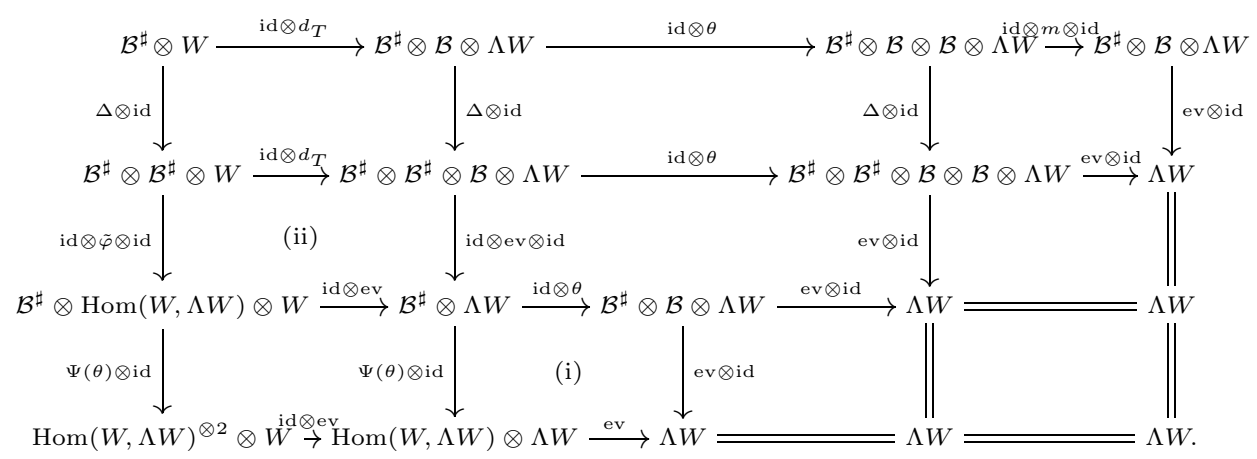

Interchanging $d_{T}$ with $\theta$ and $\tilde{\varphi}$ with $\Psi(\theta)$ and interchanging (i) with (ii) in the above diagram, we obtain the equality of the second summands of both compositions.

To see that $\Psi$ preserves brackets, let $\theta_{1}, \theta_{2} \in \operatorname{Der}_{\mathcal{B}}(\mathcal{B} \otimes \Lambda W)$ be derivations. Recall that [, ] is the bracket in $\operatorname{Der}_{\mathcal{B}}^{*}(\mathcal{B} \otimes \Lambda W)$ and $\{$,$\} is the bracket in$ $\mathcal{H} \operatorname{om}\left(\mathcal{B}^{\sharp}, \operatorname{Der}(\Lambda W)\right)$. Then, given $\beta \in \mathcal{B}^{\sharp}$ and $w \in W$, both $\left\{\Psi\left(\theta_{1}\right), \Psi\left(\theta_{2}\right)\right\}(\beta)(w)$ and $\left[\Psi\left(\theta_{1}\right), \Psi\left(\theta_{2}\right)\right](\beta)(w)$ split into two summands. We write the first summands arising from $\left\{\Psi\left(\theta_{1}\right), \Psi\left(\theta_{2}\right)\right\}$ and $\left[\Psi\left(\theta_{1}\right), \Psi\left(\theta_{2}\right)\right]$ as the upper and lower compositions, respectively, in the following diagram:

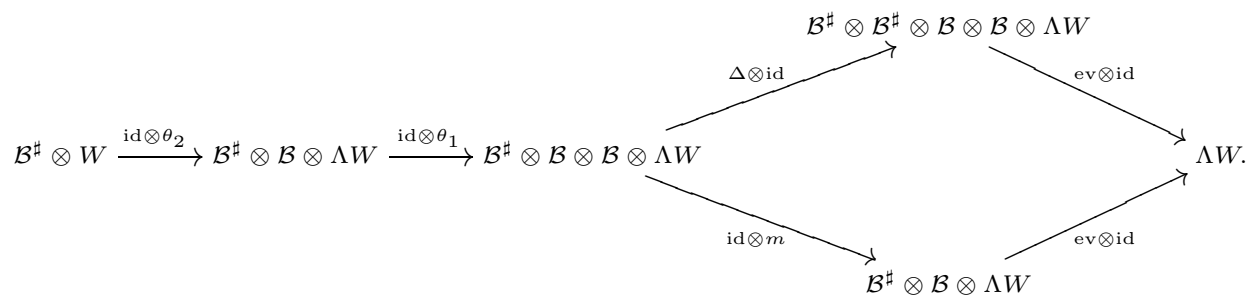

The compositions agree by the commutativity of the first diagram in (77). Similarly, the second summands in these brackets agree as may be seen by interchanging $\theta_{1}$ and $\theta_{2}$.

A proof, given in terms of elements, goes as follows. Here we work modulo signs and summations for convenience. Suppose $\theta_{1}(w)=b_{1} w_{1}, \theta_{2}(w)=b_{2} w_{2}$, $\theta_{1}\left(w_{2}\right)=b_{1}^{\prime} w_{1}^{\prime}$ and $\theta_{2}\left(w_{1}\right)=b_{2}^{\prime} w_{2}^{\prime}$. Then, on the one hand,

$$
\begin{aligned}
\left\{\Psi\left(\theta_{1}\right), \Psi\left(\theta_{2}\right)\right\}(\beta)(w)= & \sum_{j}\left[\Psi\left(\theta_{1}\right)\left(\beta_{j}^{\prime}\right), \Psi\left(\theta_{2}\right)\left(\beta_{j}^{\prime \prime}\right)\right](w) \\
= & \sum_{j}\left(\Psi\left(\theta_{1}\right)\left(\beta_{j}^{\prime}\right) \circ \Psi\left(\theta_{2}\right)\left(\beta_{j}^{\prime \prime}\right)(w)\right. \\
& \left. \pm \Psi\left(\theta_{2}\right)\left(\beta_{j}^{\prime \prime}\right) \circ \Psi\left(\theta_{1}\right)\left(\beta_{j}^{\prime}\right)(w)\right) \\
= & \sum_{j} \beta_{j}^{\prime \prime}\left(b_{2}\right) \beta_{j}^{\prime}\left(b_{1}^{\prime}\right) w_{1}^{\prime} \pm \beta_{j}^{\prime}\left(b_{1}\right) \beta_{j}^{\prime \prime}\left(b_{2}^{\prime}\right) w_{2}^{\prime} .
\end{aligned}
$$


On the other hand,

$$
\begin{aligned}
\Psi\left(\left[\theta_{1}, \theta_{2}\right]\right)(\beta)(w) & = \pm\left(\beta \otimes \operatorname{id}_{\Lambda W}\right)\left[\theta_{1}, \theta_{2}\right](w) \\
& = \pm\left(\beta \otimes \operatorname{id}_{\Lambda W}\right)\left(\theta_{1} \circ \theta_{2} \pm \theta_{2} \circ \theta_{1}\right)(w) \\
& = \pm\left(\beta \otimes \operatorname{id}_{\Lambda W}\right)\left(b_{2} b_{1}^{\prime} w_{1}^{\prime} \pm b_{1} b_{2}^{\prime} w_{2}^{\prime}\right) \\
& = \pm \beta\left(b_{2} b_{1}^{\prime}\right) w_{1}^{\prime} \pm \beta\left(b_{1} b_{2}^{\prime}\right) w_{2}^{\prime},
\end{aligned}
$$

and both expressions agree, again by the commutativity of the first diagram in (77).

We can now deduce our main result:

Proof of Theorem 1. Let $p: E \rightarrow B$ be a fibration of simply connected CW complexes with $B$ and the fibre $F$ finite. Let $h: B \rightarrow \operatorname{Baut}_{1}(F)$ be the classifying map. We choose a relative minimal model $I: \mathcal{B}, d_{B} \rightarrow \mathcal{B} \otimes \Lambda W, D$ with $\mathcal{B}$ finitedimensional and the twisted part of $D$ satisfying Lemma 2.4. By Theorem 2.5, the map $\Psi$ is a DG Lie algebra isomorphism. The result now follows from Propositions 2.1 , 2.2 and 2.3 .

Remark 2.6. Given a fibration $p: E \rightarrow B$ as hypothesized in Theorem 1 choose a base-point for $E$ and let aut $t_{1}^{*}(p)$ denote the space of base-point-preserving fibre homotopy equivalences of $p$. The above arguments apply to give a Quillen model of the form $\overline{\mathcal{D e r}}_{\mathcal{B}}(\mathcal{B} \otimes \Lambda W), \mathcal{D}$ for the space $\operatorname{Baut}_{1}^{*}(p)$. Here

$$
\overline{\mathcal{D e r}}_{\mathcal{B}}(\mathcal{B} \otimes \Lambda W)=\left\{\theta \in \mathcal{D e r}_{\mathcal{B}}(\mathcal{B} \otimes \Lambda W) \mid \theta(w) \in(\mathcal{B} \otimes \Lambda W)_{+}\right\} .
$$

The differential $\mathcal{D}$ and bracket [, ] are those induced from $\operatorname{Der}_{\mathcal{B}}(\mathcal{B} \otimes \Lambda W), \mathcal{D}$. We omit the details.

\section{Two PRoblems on FIBREWISE SELF-EQUIVALENCES}

Let $p: E \rightarrow B$ be a fibration of simply connected CW complexes with $B$ and the fibre $F$ finite. The assignment $p \mapsto \operatorname{Baut}_{1}(p)_{\mathbb{Q}}$ induces a function

$$
\frac{\{\text { fibrations } p: E \rightarrow B \text { with fibre } F\}}{\simeq_{\text {fhe }}} \rightarrow \frac{\left\{\text { classifying spaces } \operatorname{Baut}_{1}(p)\right\}}{\simeq_{\mathbb{Q}}},
$$

where $\simeq_{\text {fhe }}$ denotes fibre-homotopy equivalence and $\simeq_{\mathbb{Q}}$ rational equivalence. The first general problem we propose is the following analogue of the gauge group classification problem mentioned in the introduction.

Problem 3.1. Classify the distinct rational homotopy types in the image of the function (8) for fixed fibre $F$ and base $B$.

When the rationalization $Y_{\mathbb{Q}}$ of a nilpotent space $Y$ is an $H$-space we say that $Y$ is a rational $H$-space. When $\operatorname{Baut}_{1}(p)$ is a rational $H$-space, the monoid $\operatorname{aut}_{1}(p)$ is then a double loop-space after rationalization and, in particular, rationally $H$ homotopy commutative (cf. [19]). It is natural to consider:

Problem 3.2. Determine conditions on $p: E \rightarrow B$ that ensure $\operatorname{Baut}_{1}(p)$ is a rational $H$-space. 
The function space identity for $\operatorname{Baut}_{1}(p)$ (Proposition 2.1) leads to a class of familiar examples for which both problems admit the simplest possible solution. The basic idea has appeared in many places (see [4, 10, 15]). We have:

Theorem 3.3. Let $F$ be a $C W$ complex of finite type and $B$ a simply connected finite $C W$ complex. Suppose $\operatorname{Baut}_{1}(F)$ is a rational $H$-space. Then the the classifying spaces Baut $_{1}(p)$ for fibrations $p$ with fibre $F$ and base $B$ are all rational $H$-spaces and pairwise rationally homotopy equivalent.

Proof. Given an F-fibration $p: E \rightarrow B$, as hypothesized, we have

$$
\operatorname{Baut}_{1}(p)_{\mathbb{Q}} \simeq \widetilde{\operatorname{map}}\left(B, \operatorname{Baut}_{1}(F) ; h\right)_{\mathbb{Q}} \simeq \widetilde{\operatorname{map}}\left(B, \operatorname{Baut}_{1}(F)_{\mathbb{Q}} ; h_{\mathbb{Q}}\right),
$$

where the first equivalence is (1) and the second is [14, Th. 3.11]. Here $h_{\mathbb{Q}}$ denotes $h$ composed with a rationalization $r: \operatorname{Baut}_{1}(F) \rightarrow \operatorname{Baut}_{1}(F)_{\mathbb{Q}}$. Now a rationalized $H$-space is homotopy equivalent to a loop-space ([19, Lem. 0.1]) and so, in particular, admits a homotopy inverse. It follows that the path-components of $\widetilde{\operatorname{map}}\left(B, \operatorname{Baut}_{1}(F)_{\mathbb{Q}}\right)$ are each homotopy equivalent to $\widetilde{\operatorname{map}}\left(B, \operatorname{Baut}_{1}(F)_{\mathbb{Q}} ; 0\right)$, the component of the null map. $\operatorname{Since} \operatorname{map}\left(B, \operatorname{Baut}_{1}(F)_{\mathbb{Q}} ; 0\right)$ is an $H$-space with pointwise multiplication, so is its universal cover.

Remarks 3.4. (1) The famous Halperin conjecture [8, 39.1], affirmed in many cases, implies that $\operatorname{Baut}_{1}(F)$ is a rational $H$-space whenever $F$ is an elliptic CW complex with $H^{*}(F ; \mathbb{Q})$ evenly graded. See $[9$, Ex. 2.6] for a discussion in this context. Theorem 3.3 applies, for example, when $F$ has truncated polynomial rational cohomology or $F=G / H$ for $H \subseteq G$ equal rank, compact, connected Lie pairs [21].

(2) On the other hand, if $G$ is a topological group, then $\operatorname{Baut}_{1}(G)$ is rarely a rational $H$-space: in fact, only when $\pi_{*}(G) \otimes \mathbb{Q}$ is concentrated in a single degree. Problems 3.1 and 3.2 are thus already interesting when restricted to principal $G$ bundles over a fixed base $B$.

(3) A similar argument shows that the classifying spaces $B \mathcal{G}(p)$ for gauge groups of $G$-principal bundles $p: E \rightarrow B$ are pairwise rationally homotopy equivalent and each is a rational $H$-space. The assumption that $B$ is simply connected is no longer required since the identity $B \mathcal{G}(p) \simeq \operatorname{map}(B, B G ; h)$ holds without this restriction. It is classical that $B G$ is a rational $H$-space for $G$ connected. This result for $B \mathcal{G}(p)$ holds, in fact, for $B$ any compact metric space [15, Th. D]. Theorem 3.3 and (1) above also extend to spaces $B$ without CW structure by [20, Th. 2].

We give a sample of results on Problems 3.1 and 3.2 in the some cases not covered by Theorem 3.3. Fix $F$ and $B$ both finite and simply connected. Let $\mathcal{B}, d_{B}$ be a finite model for $B$ and $\Lambda W, d_{W}$ the Sullivan minimal model for $F$. The relative minimal models for $F$-fibrations over $B$ are of the form

$$
I: \mathcal{B}, d_{B} \rightarrow \mathcal{B} \otimes \Lambda W, D
$$

Recall that the differential $D$ extends $d_{B}$ and satisfies the nilpotence condition whereby $W=\bigoplus_{i>0} W_{i}$ and $D\left(W_{i}\right) \subseteq \mathcal{B} \otimes \Lambda W_{<i}$ (see [8, Sec. 14]). We are led to consider the possible differentials $D$ on $\mathcal{B} \otimes \Lambda W$. These differentials give rise, in turn, to differentials $\mathcal{D}$ for the graded Lie algebra $\operatorname{Der}_{\mathcal{B}}(\mathcal{B} \otimes \Lambda W)$. We will make use of the following consequence of Theorem 1 
Corollary 3.5. Let $B$ and $F$ be finite, simply connected $C W$ complexes.

(A) Given F-fibrations $p$ and $p^{\prime}$ over $B$, then $\operatorname{Baut}_{1}(p) \simeq_{\mathbb{Q}} \operatorname{Baut}_{1}\left(p^{\prime}\right)$ if and only if there is a quasi-isomorphism

$$
\psi: \operatorname{Der}_{\mathcal{B}}(\mathcal{B} \otimes \Lambda W), \mathcal{D} \rightarrow \mathcal{D} \operatorname{er}_{\mathcal{B}}(\mathcal{B} \otimes \Lambda W), \mathcal{D}^{\prime} .
$$

(B) The following are equivalent.

(i) $\operatorname{Baut}_{1}(p)$ is a rational H-space.

(ii) $\operatorname{Der}_{\mathcal{B}}(\mathcal{B} \otimes \Lambda W), \mathcal{D}$ is quasi-isomorphic to an abelian $D G$ Lie algebra.

(iii) $\mathcal{C}^{*}\left(\operatorname{Der}_{\mathcal{B}}(\mathcal{B} \otimes \Lambda W), \mathcal{D}\right)$ is quasi-isomorphic to a $D G$ algebra with trivial differential.

3.1. The classification problem for principal bundles. Problem 3.1 is quite complicated for general $F$ and $B$. We restrict attention to principal $G$-bundles $p: E \rightarrow B$ for fixed $G$ and $B$. This ensures that the differential $D$ is pure in the sense that $D(W) \subseteq \mathcal{B}[8$, Sec.15.f]. When $D$ is pure, the differential $\mathcal{D}$ reduces to $\mathcal{D}(\theta)=D \circ \theta$ for $\theta \in \mathcal{D} \operatorname{er}_{\mathcal{B}}(\mathcal{B} \otimes \Lambda W)$.

Suppose now that $W \cong \pi_{*}(G) \otimes \mathbb{Q}$ is finite-dimensional. Let $n$ denote the maximal nontrivial degree; i.e., $\pi_{n}(G) \otimes \mathbb{Q} \neq 0$ and $\pi_{k}(G) \otimes \mathbb{Q}=0$ for $k>n$. Here is one general situation when nonequivalent bundles yield the same rational homotopy type for the classifying space:

Lemma 3.6. Let $p$ and $p^{\prime}$ be principal $G$-bundles over $B$ with differentials $D$ and $D^{\prime}$ in their relative minimal models, respectively. If $D=D^{\prime}$ on $W_{<n}$, where $n$ is the maximal nontrivial degree of $W$, then $\operatorname{Baut}_{1}(p) \simeq_{\mathbb{Q}} \operatorname{Baut}_{1}\left(p^{\prime}\right)$.

Proof. The identity map for $\mathcal{B} \otimes \Lambda W$ induces a quasi-isomorphism of DG Lie algebras $\mathcal{D e r}_{\mathcal{B}}(\mathcal{B} \otimes \Lambda W), \mathcal{D} \rightarrow \mathcal{D e r}_{B}(\mathcal{B} \otimes \Lambda W), \mathcal{D}^{\prime}$ with these hypotheses.

Now suppose $G$ is a simply connected topological group of the homotopy type of a finite CW complex. This class includes the simply connected Lie groups and ensures that $W=\pi_{*}(G) \otimes \mathbb{Q}$ is oddly-graded and finite-dimensional. The principal $G$-bundles over $B$ are represented by homotopy classes $[B, B G]$. Rationalizing, this set becomes $\operatorname{Hom}^{-1}\left(W, H^{*}(B ; \mathbb{Q})\right)$, which corresponds, in turn, to the set of pure differentials $D$ on $\mathcal{B} \otimes \Lambda W$. When $W$ is concentrated in a single degree, Lemma 3.6 implies that the classifying spaces $\operatorname{Baut}_{1}(p)$ are all of the same rational homotopy type. When $W$ is concentrated in 2 or more degrees, the number of rational homotopy types depends on the cohomology of $B$. Here is a first example.

Proposition 3.7. The principal $S U(3)$-bundles $p: E \rightarrow B$ over a simply connected, finite $C W$ complex $B$ give rise to either one or two rational homotopy types for $\operatorname{Baut}_{1}(p)$ depending, respectively, on whether $H^{4}(B ; \mathbb{Q})$ is trivial or not.

Proof. The relative minimal model for such a bundle $p$ is of the form

$$
\mathcal{B}, d_{B} \rightarrow \mathcal{B} \otimes \Lambda\left(s_{3}, s_{5}\right), D,
$$

where $\left|s_{i}\right|=i$. If $H^{4}(B ; \mathbb{Q})=0$, then $D\left(s_{3}\right)=0$ and so the result follows from Lemma 3.6 .

If $H^{4}(B ; \mathbb{Q}) \neq 0$, then we may set $D\left(s_{3}\right)=\chi_{4}$ for $\left\langle\chi_{4}\right\rangle \neq 0 \in H^{4}(B ; \mathbb{Q})$ and $D\left(s_{5}\right)=0$. We claim that

$$
\operatorname{rank} H_{2}\left(\mathcal{D e r}_{\mathcal{B}}(\mathcal{B} \otimes \Lambda W), \mathcal{D}\right)=\operatorname{rank} H^{3}(B ; \mathbb{Q})
$$

while

$$
\operatorname{rank} H_{2}\left(\operatorname{Der}_{\mathcal{B}}(\mathcal{B} \otimes \Lambda W), 0\right)=\operatorname{rank} H^{3}(B ; \mathbb{Q})+1
$$


For the second equation, observe that, generally,

$$
H_{n}\left(\mathcal{D e r}_{\mathcal{B}}(\mathcal{B} \otimes \Lambda W), 0\right)=\operatorname{Hom}^{n}\left(W, H^{*}(B ; \mathbb{Q})\right) .
$$

For the first equation, suppose that the derivation $\left(s_{5}, x_{3}+q s_{3}\right)$ is a $\mathcal{D}$-cycle. Here we write $(w, \chi)$ for the derivation in $\operatorname{Der}_{B}(\mathcal{B} \otimes \Lambda W)$ carrying $w$ to $\chi$ and vanishing on a complementary basis for $w \in W$. Then

$$
0=\mathcal{D}\left(s_{5}, x_{3}+q s_{3}\right)=\left(s_{5}, d_{B}\left(x_{3}\right)+q \chi_{4}\right) .
$$

Thus, since $\left\langle\chi_{4}\right\rangle \neq 0$, we must have $q=0$.

Finally, if $D^{\prime}$ is another pure differential with $D^{\prime}\left(s_{3}\right)=\chi_{4}^{\prime}$ another nonbounding cycle, then the map sending $\chi_{4} \rightarrow \chi_{4}^{\prime}$ and fixing the other generators of $\mathcal{B} \otimes \Lambda W$ induces a quasi-isomorphism $\mathcal{D e r}_{\mathcal{B}}(\mathcal{B} \otimes \Lambda W), \mathcal{D} \rightarrow \operatorname{Der}_{B}(\mathcal{B} \otimes \Lambda W), \mathcal{D}^{\prime}$.

We give a complete result when $B$ has very simple rational cohomology. Given a fibration $p: E \rightarrow B$ with relative minimal model $\mathcal{B}, d_{B} \rightarrow \mathcal{B} \otimes \Lambda W, D$, and pure differential $D$, define a nonnegative integer

$$
n(p)= \begin{cases}\min \left\{k \mid D\left(W_{k}\right) \neq 0\right\} & \text { for } D(W) \neq 0 \\ n & \text { for } D(W)=0 .\end{cases}
$$

Example 1.1 is contained in the following:

Proposition 3.8. Let $B$ be a simply connected finite $C W$ complex with $H^{*}(B ; \mathbb{Q})$ generated in a single even degree. Let $G$ be a simply connected topological group with the homotopy type of a finite $C W$ complex. Given two principal $G$-bundles $p, q$ over $B$ we have

$$
\operatorname{Baut}_{1}(p) \simeq_{\mathbb{Q}} \operatorname{Baut}_{1}(q) \text { if and only if } n(p)=n(q) .
$$

Proof. Since $B$ is finite, $H^{*}(B ; \mathbb{Q})$ is a truncated polynomial algebra. A finite DG algebra model for $B$ is of the form $\mathcal{B}=\Lambda(x) /\left\langle x^{m+1}\right\rangle$ for some $x$ of even degree, say $2 k$. Given a principal $G$-bundle $p: E \rightarrow B$, the relative minimal model for $p$ is thus of the form

$$
\mathcal{B}, 0 \rightarrow \mathcal{B} \otimes \Lambda W, D
$$

with $D(x)=0$ and $D(w)=\chi(w)$ for $\chi(w)$ a cycle in $\mathcal{B}$ of degree $|w|+1$. Note that if $\chi(w) \neq 0$, then $\chi(w)=q x^{j}$ for some $j$ and $q \in \mathbb{Q}$. We may thus write

$$
W=\mathbb{Q}\left(w_{1}, \ldots, w_{s}\right) \oplus W^{\prime},
$$

where (i) the $w_{i}$ are of strictly increasing odd degrees $q_{i}$, (ii) $D\left(w_{i}\right)=x^{n_{i}}$ for some $n_{i} \leq n$ and (iii) $D\left(W^{\prime}\right)=0$. Define a second differential $D^{\prime}$ by setting $D^{\prime}\left(w_{1}\right)=x^{n_{1}}$, $D^{\prime}\left(w_{i}\right)=0$ for $i \geq 2$ and $D^{\prime}\left(W^{\prime}\right)=0$. Define

$$
\psi: \mathcal{B} \otimes \Lambda W, D^{\prime} \rightarrow \mathcal{B} \otimes \Lambda W, D
$$

by $\psi\left(w_{1}\right)=w_{1}, \psi\left(w_{i}\right)=w_{i}-x^{n_{i}-n_{1}} w_{1}$ for $i \geq 2$ and $\psi$ the identity on $\Lambda W^{\prime}$ and $\mathcal{B}$. The map $\psi$ induces a quasi-isomorphism $\operatorname{Der}_{\mathcal{B}}(\mathcal{B} \otimes \Lambda W), \mathcal{D}^{\prime} \rightarrow \mathcal{D}_{\operatorname{er}_{B}^{*}}(\mathcal{B} \otimes \Lambda W), \mathcal{D}$.

Suppose now that $D$ and $D^{\prime}$ are differentials on $\mathcal{B} \otimes \Lambda W$ given, on $W$, as $D=$ $\left(w, x^{k}\right)$ and $D^{\prime}=\left(w^{\prime}, x^{l}\right)$ for $k, l \leq m$. Suppose $|w| \neq\left|w^{\prime}\right|$, say $|w|<\left|w^{\prime}\right|$. Let $n$ be the maximal nontrivial degree of $W$. Then we see that

$$
\operatorname{rank} H_{n-|w|}\left(\mathcal{D e r}_{\mathcal{B}}(\mathcal{B} \otimes \Lambda W ; D)\right)<\operatorname{rank} H_{n-|w|}\left(\mathcal{D e r}_{\mathcal{B}}\left(\mathcal{B} \otimes \Lambda V ; D^{\prime}\right)\right)
$$

since $\left(w_{n}, w\right)$ is a nonbounding $D^{\prime}$-cycle but is not a $D$-cycle for $w_{n} \in W_{n}$.

Applying the functor $\mathcal{C}^{*}$, we can identify the rational homotopy types represented by $\operatorname{Baut}_{1}(p)$ for fixed $F$ and $B$ in special cases. We give a representative example: 
Example 3.9. We consider the case $F=S^{5} \times S^{7}$ and $B=S^{3} \times S^{3}$. A finitedimensional DG algebra model $\mathcal{B}=\Lambda\left(x_{3}, y_{3}\right)$ in this case. The relative minimal model for such a fibration is of the form

$$
\mathcal{B}, 0 \rightarrow \mathcal{B} \otimes \Lambda\left(s_{5}, s_{7}\right), D .
$$

Up to rational fibre homotopy equivalence, there are two possibilities for $D$. Either $D=0$ or $D\left(s_{5}\right)=x_{3} y_{3}$ and $D\left(s_{7}\right)=0$. In both cases, we have the following basis for $\operatorname{Der}_{\mathcal{B}}\left(\mathcal{B} \otimes \Lambda\left(s_{5}, s_{7}\right)\right)$ :

$$
\begin{array}{ccc}
\alpha=\left(s_{7}, x_{3} y_{3}\right), & \beta_{1}=\left(s_{5}, x_{3}\right), \quad \beta_{2}=\left(s_{5}, y_{3}\right), \quad \beta_{3}=\left(s_{7}, s_{5}\right), \\
\gamma_{1}=\left(s_{7}, x_{3}\right), & \gamma_{2}=\left(s_{7}, y_{3}\right), \quad \eta=\left(s_{5}, 1\right), \quad \phi=\left(s_{7}, 1\right) .
\end{array}
$$

Here $|\alpha|=1,\left|\beta_{i}\right|=2,\left|\gamma_{i}\right|=4,|\eta|=5$ and $|\phi|=7$. Thus $\mathcal{C}^{*}\left(\mathcal{D e r}_{\mathcal{B}}\left(\mathcal{B} \otimes \Lambda\left(s_{5}, s_{7}\right)\right), \mathcal{D}\right)$ is a DG algebra of the form

$$
\Lambda\left(a, b_{1}, b_{2}, b_{3}, c_{1}, c_{2}, e, f\right), d,
$$

where $|a|=2,\left|b_{i}\right|=3,\left|c_{i}\right|=5,|e|=6$ and $|f|=8$.

When $D=0$, the induced differential $\mathcal{D}=0$, and so the differential $d$ is given, in this case, by

$$
d(a)=d\left(b_{1}\right)=d\left(b_{2}\right)=d\left(b_{3}\right)=d(e)=0, d\left(c_{1}\right)=b_{3} b_{1}, d\left(c_{2}\right)=b_{3} b_{2}, d(f)=b_{3} c .
$$

It is easy to see that $\operatorname{Baut}_{1}(p)$ is not a formal space in this case.

When $D \neq 0$ the differential $\mathcal{D}$ is trivial except that $\mathcal{D}\left(\beta_{3}\right)=\alpha$. Removing this linear relation from $\mathcal{D e r}_{\mathcal{B}}\left(\mathcal{B} \otimes \Lambda\left(s_{5}, s_{7}\right)\right)$, $\mathcal{D}$ we see that

$$
\operatorname{Baut}_{1}(p) \simeq K\left(\mathbb{Q}^{2}, 3\right) \times K\left(\mathbb{Q}^{2}, 5\right) \times K(\mathbb{Q}, 6) \times K(\mathbb{Q}, 8)
$$

in this case.

3.2. When is $\operatorname{Baut}_{1}(p)$ a rational $H$-space? We give some sample results addressing this question. By Theorem 3.3 , Baut ${ }_{1}(p)$ is a rational $H$-space when $\operatorname{Baut}_{1}(F)$ is one. In addition to the spaces mentioned in Remark 3.4 (1), this occurs when $\pi_{*}(F) \otimes \mathbb{Q}$ is concentrated in a single degree. When $\pi_{*}(F) \otimes \mathbb{Q}$ is concentrated in two degrees, we have the following result. Recall that a simply connected CW complex $X$ is coformal if $X$ admits a Quillen model with vanishing differential or, equivalently, if the minimal Sullivan model for $X$ has purely quadratic differential.

Proposition 3.10. Let $p: E \rightarrow B$ be a fibration of simply connected $C W$ complexes with fibre $F$ and base $B$ finite. Suppose $\pi_{*}(F) \otimes \mathbb{Q}$ is concentrated in two degrees. Then $\operatorname{Baut}_{1}(p)$ is a coformal space and $\operatorname{Baut}_{1}(p)$ is a rational H-space if and only if $\operatorname{aut}_{1}(p)$ is rationally H-homotopy commutative.

Proof. In this case, the DG Lie algebra $\mathcal{D e r}_{\mathcal{B}}(\mathcal{B} \otimes \Lambda W), D$ only admits iterated brackets of length two. It is an easy exercise now to show that a minimal model for $C^{*}\left(\mathcal{D e r}_{\mathcal{B}}(\mathcal{B} \otimes \Lambda W), D\right)$ has purely quadratic differential. In general, if $X$ is a coformal space, then $X$ is a rational $H$-space if and only if the $\Omega X$ is rationally $H$-homotopy commutative.

Focusing on a concrete example, we have:

Proposition 3.11. Let $p: E \rightarrow \mathbb{C} P^{m}$ be a principal $S U(n)$-bundle. Then $\operatorname{Baut}_{1}(p)$ is a rational $H$-space if and only if either $n=1,2$ or $n=3$ and the characteristic class $\chi_{1}(p) \neq 0 \in H^{4}\left(\mathbb{C} P^{m} ; \mathbb{Q}\right)$. 
Proof. When $n=1$ the fibre is contractible and so $\operatorname{aut}_{1}(p)$ is also. For $n=2$, the result is Theorem 3.3. as mentioned above. For $n=3$, observe that the induced bracket in $\mathcal{D e r}_{\mathcal{B}}\left(\mathcal{B} \otimes \Lambda\left(s_{3}, s_{5}\right)\right), \mathcal{D}$ is nontrivial if and only if the derivation $\left(s_{5}, s_{3}\right)$ is a $\mathcal{D}$-cycle or, equivalently, $D\left(s_{3}\right)=0$. For $n>3$, we invoke the proof of Proposition 3.8. There we showed that, in the relative minimal model $\mathcal{B}, d_{B} \rightarrow \mathcal{B} \otimes \Lambda\left(s_{3}, \ldots, s_{2 n-1}\right)$, we may assume $D\left(s_{2 i+1}\right) \neq 0$ for at most one $i \geq 1$. Thus $D\left(s_{2 i-1}\right)=0$ for some $2 \leq i<n$. The bracket

$$
\left[\left(s_{2 n-1}, s_{2 i-1}\right),\left(s_{2 i-1}, 1\right)\right]=\left(s_{2 n-1}, 1\right)
$$

is then a nonbounding bracket of $\mathcal{D}$-cycles. Thus $\pi_{*}\left(\operatorname{aut}_{1}(p)\right) \otimes \mathbb{Q}$ has a nontrivial Samelson bracket for $n>3$.

Finally, we give a connection between Massey products in the rational cohomology of $E$ and nontrivial brackets in the Samelson Lie algebra of $\operatorname{aut}_{1}(p)$. Let $p: E \rightarrow B$ be a principal $G$-bundle where, as usual, $B$ is a finite, simply connected CW complex and $G$ is a simply connected topological group of the homotopy type of a finite CW complex. Let $j: G \rightarrow E$ denote the fibre inclusion. As in Example 1.2 . we consider the map

$$
J=\operatorname{Hur}_{\mathbb{Q}} \circ j_{\sharp}: \pi_{*}(G) \rightarrow H_{*}(E ; \mathbb{Q}),
$$

where $\operatorname{Hur}_{\mathbb{Q}}: \pi_{*}(E) \rightarrow H_{*}(E ; \mathbb{Q})$ is the rational Hurewicz map. We may identify the image of $J$ in terms of the relative minimal model $\mathcal{B}, d_{B} \rightarrow \mathcal{B} \otimes \Lambda W, D$ for $p$. Recall that $D$ is pure. Define

$$
\operatorname{ker}\left[D_{W}\right]=\left\{w \in W \mid D(w) \text { is cohomologous to zero in } \mathcal{B} \otimes \Lambda W_{<|w|}, D\right\} .
$$

Note that $\operatorname{ker}\left[D_{W}\right]$ consists of $D$-cycles of the Sullivan model $\mathcal{B} \otimes \Lambda W, D$ for $E$ and thus determines a subspace $K \subseteq H^{*}(E ; \mathbb{Q})$. Then we have

$$
\operatorname{Image}(J) \cong \operatorname{Hom}(K ; \mathbb{Q}) \text {. }
$$

To see this, observe that an element $w \in \operatorname{ker}\left[D_{W}\right]$ corresponds, after a change of basis, to a nonbounding primitive $D$-cycle of generator of the Sullivan model of $E$ and thus to an element in the image of the rational Hurewicz map. In particular, $J=0$ if and only if $\operatorname{ker}\left[D_{W}\right]=0$.

Lemma 3.12. Let $p$ have a relative minimal model as described above. If $\operatorname{Baut}_{1}(p)$ is a rational H-space, then

$$
\operatorname{Image}(J) \cap H_{<n}(E ; \mathbb{Q})=0,
$$

where $n$ is the maximal degree for which $W$ is nontrivial.

Proof. Using (9), suppose $D(w)=D(\chi)$ for some $w \in W_{q}$ with $q<n$ and $\chi \in$ $\mathcal{B} \otimes \Lambda W_{<q}$. Then we may perform a basis change: Define $\varphi: \mathcal{B} \otimes \Lambda W \rightarrow \mathcal{B} \otimes \Lambda W$ by sending $w \rightarrow w-\chi$ and fixing the other elements of $W$ and all of $\mathcal{B}$. Define $D^{\prime}$ by setting $D^{\prime}(w)=0$ and $D^{\prime}=D$ on a complement of $\mathbb{Q}(w) \subseteq W$ and on $\mathcal{B}$. The result is a DG map inducing a quasi-isomorphism $\operatorname{Der}_{\mathcal{B}}(\mathcal{B} \otimes \Lambda W), \mathcal{D}^{\prime} \rightarrow \mathcal{D e r}_{\mathcal{B}}(\mathcal{B} \otimes \Lambda W), \mathcal{D}$. In the former DG Lie algebra, we have

$$
\left[\left(w_{n}, w\right),(w, 1)\right]=\left(w_{n}, 1\right),
$$

a nonbounding bracket of $\mathcal{D}^{\prime}$-cycles for $w_{n} \in W_{n}$. 
To discuss Massey products in $H^{*}(E ; \mathbb{Q})$ we need $\mathcal{B} \otimes \Lambda W, D$ to be a minimal Sullivan model for $E$. By [13, Prop. 4.12], this is equivalent to requiring $p: E \rightarrow B$ to be Whitehead trivial, i.e., having a trivial linking homomorphism in its long exact rational homotopy sequence. In the setting of principal bundles, Whitehead triviality is equivalent to the assumption that the differential $D$ satisfies

$$
D(W) \subseteq H^{+}(B ; \mathbb{Q}) \cdot H^{+}(B ; \mathbb{Q}) .
$$

We recall the characterization of formality in terms of Massey products in [6]. Let $\Lambda V, d$ be a simply connected DG algebra. Let $C \subseteq V$ denote the cycles of $V$. By a Massey product in $H^{*}(\Lambda V, d)$ we mean a nonzero element represented by a cycle in $I\left(V^{\prime}\right) \subseteq \Lambda V$, where $V^{\prime}$ is some vector space complement to $C \subseteq V$ and $I\left(V^{\prime}\right) \subseteq \Lambda V$ is the ideal generated by $V^{\prime}$. By [6. Lem. 4.3], $\Lambda V, d$ is formal if and only if all Massey products vanish. The following result implies Example 1.2,

Theorem 3.13. Let $B$ be a finite simply connected $C W$ complex and $G$ a simply connected topological group of the homotopy type of a finite $C W$ complex. Let $p: E \rightarrow B$ be a Whitehead trivial, principal $G$-bundle. Let $n$ be the maximal degree for which $\pi_{n}(G) \otimes \mathbb{Q} \neq 0$. Suppose $(i) J: \pi_{*}(G) \rightarrow H_{*}(E ; \mathbb{Q})$ vanishes in degrees $<n$ and $\left(\right.$ ii) $H^{*}(E ; \mathbb{Q})$ has no Massey products of degree $<n$. Then $\operatorname{Baut}_{1}(p)$ is a rational $H$-space.

Proof. Since $J$ vanishes in degrees $<n$, in the minimal Sullivan model $\mathcal{B} \otimes \Lambda W, D$ for $E$ we may take $W \subseteq V^{\prime}$. Suppose $\theta$ is a cycle in $\mathcal{D e r}_{\mathcal{B}}^{q}(\mathcal{B} \otimes \Lambda W), \mathcal{D}$. Given $w \in W$, write $\theta(w)=z+m$, where $m \in I\left(V^{\prime}\right)$ and $z \in \Lambda C$. Since $\theta$ is a $\mathcal{D}$-cycle, $D(m)=0$. Thus $m=D(y)$ for some $y \in \mathcal{B} \otimes \Lambda W$. We then have $(\theta-\mathcal{D}(w, y))(w) \subseteq \mathcal{B}$. Continuing in this way for each element in a basis for $W$, we obtain that $\theta$ is homologous to a $\mathcal{D}$-cycle $\theta^{\prime}$ satisfying $\theta^{\prime}(W) \subseteq \mathcal{B}$. It follows that the inclusion

$$
\mathcal{H o m}(W, \mathcal{B}), 0 \hookrightarrow \mathcal{D e r}_{\mathcal{B}}(\mathcal{B} \otimes \Lambda W), D
$$

is a quasi-isomorphism. Since $\mathcal{H o m}(W, \mathcal{B}), 0$ is an abelian DG Lie algebra, the result follows from Corollary 3.5 (B).

\section{REFERENCES}

[1] M. F. Atiyah and R. Bott, The Yang-Mills equations over Riemann surfaces, Philos. Trans. Roy. Soc. London Ser. A 308 (1983), no. 1505, 523-615. MR702806 (85k:14006)

[2] Urtzi Buijs, Yves Félix, and Aniceto Murillo, Lie models for the components of sections of a nilpotent fibration, Trans. Amer. Math. Soc. 361 (2009), no. 10, 5601-5614. MR2515825 (2010e:55006)

[3] $L_{\infty}$ models of based mapping spaces, J. Math. Soc. Japan. 63 (2011), no. 2, 503-524. MR2793109

[4] Peter Booth, Philip Heath, Chris Morgan, and Renzo Piccinini, H-spaces of self-equivalences of fibrations and bundles, Proc. London Math. Soc. (3) 49 (1984), no. 1, 111-127. MR743373 (85k:55013)

[5] M. C. Crabb and W. A. Sutherland, Counting homotopy types of gauge groups, Proc. London Math. Soc. (3) 81 (2000), no. 3, 747-768. MR1781154(2001m:55024)

[6] Pierre Deligne, Phillip Griffiths, John Morgan and Dennis Sullivan, Real homotopy theory of Kähler manifolds, Invent. Math. 29 (1975), no. 3, 245-274. MR0382702 (52:3584)

[7] Albrecht Dold and Richard Lashof, Principal quasi-fibrations and fibre homotopy equivalence of bundles, Illinois J. Math. 3 (1959), 285-305. MR0101521 (21:331)

[8] Yves Félix, Stephen Halperin, and Jean-Claude Thomas, Rational homotopy theory, Graduate Texts in Mathematics, vol. 205, Springer-Verlag, New York, 2001. MR.1802847 (2002d:55014) 
[9] Yves Félix, Gregory Lupton, and Samuel B. Smith, The rational homotopy type of the space of self-equivalences of a fibration, Homology, Homotopy Appl. 12 (2010), no. 2, 371-400. MR2771595

[10] Yves Félix and John Oprea, Rational homotopy of gauge groups, Proc. Amer. Math. Soc. 137 (2009), no. 4, 1519-1527. MR2465678 (2009h:55011)

[11] J.-B. Gatsinzi, The homotopy Lie algebra of classifying spaces, J. Pure Appl. Alg. 120 (1997), 281-289. MR1468920 (98g:55015)

[12] Daniel Henry Gottlieb, On fibre spaces and the evaluation map, Ann. of Math. (2) 87 (1968), 42-55. MR.0221508 (36:4560)

[13] Stephen Halperin, Rational fibrations, minimal models, and fibrings of homogeneous spaces, Trans. Amer. Math. Soc. 244 (1978), 199-224. MR0515558 (58:24264)

[14] Peter Hilton, Guido Mislin, and Joe Roitberg, Localization of nilpotent groups and spaces, North-Holland Publishing Co., Amsterdam, 1975, North-Holland Mathematics Studies, No. 15. MR0478146(57:17635)

[15] John R. Klein, Claude L. Schochet, and Samuel B. Smith, Continuous trace C*-algebras, gauge groups and rationalization, J. Topol. Anal. 1 (2009), no. 3, 261-288. MR2574026 (2010m:55011)

[16] Akira Kono, A note on the homotopy type of certain gauge groups, Proc. Roy. Soc. Edinburgh Sect. A 117 (1991), no. 3-4, 295-297. MR1103296 (92b:55005)

[17] Akira Kono and Shuichi Tsukuda, 4-manifolds $X$ over BSU(2) and the corresponding homotopy types $\operatorname{Map}(X, B \mathrm{SU}(2))$, J. Pure Appl. Algebra 151 (2000), no. 3, 227-237. MR.1776430 (2001k:55020)

[18] Peter May, Classifying spaces and fibrations, Mem. Amer. Math. Soc., 1 (1975), no. 155. MR0370579(51:6806)

[19] Hans Scheerer, On rationalized $H$ - and co-H-spaces. With an appendix on decomposable $H$ and co-H-spaces, Manuscripta Math. 51 (1985), no. 1-3, 63-87. MR788673 (86m:55016)

[20] Claude L. Schochet and Samuel B. Smith, Localization of grouplike function and section spaces with compact domain, Homotopy theory of function spaces and related topics, Contemp. Math., vol. 519, Amer. Math. Soc., Providence, RI, 2010, pp. 189-202. MR2648714 (2011f:55023)

[21] H. Shiga and M. Tezuka, Rational fibrations, homogeneous spaces with positive Euler characteristics and Jacobians, Ann. Inst. Fourier (Grenoble) 37 (1987), no. 1, 81-106. MR894562 (89g:55019)

[22] Dennis Sullivan, Infinitesimal computations in topology, Inst. Hautes Études Sci. Publ. Math. (1977), no. 47, 269-331 (1978). MR0646078 (58:31119)

[23] Daniel Tanré, Homotopie rationnelle: Modèles de Chen, Quillen, Sullivan. Lecture Notes in Mathematics 1025, Springer-Verlag, Berlin, 1983. MR764769 (86b:55010)

[24] Shuichi Tsukuda, A remark on the homotopy type of the classifying space of certain gauge groups, J. Math. Kyoto Univ. 36 (1996), no. 1, 123-128. MR1381543 (97h:55017)

Departament d'Àlgebra i Geometria, Universitat de Barcelona, Gran Via de les Corts Catalanes, 585, 08007 Barcelona, Spain

E-mail address: ubuijs@ub.edu

Department of Mathematics, Saint Joseph's University, Philadelphia, Pennsylvania 19131

E-mail address: smith@sju.edu 